\title{
Osteotomía percutánea de la falange distal del hallux. Indicaciones y técnica
}

\author{
Juan Manuel Yañez Arauz, ‘ Juan Martín Yañez Arauz, " Verónica Montiel Terrón,"* Verónica Machado," \\ Matías Alfonso," Carlos Villas-Tomé ${ }^{* *}$ \\ "Sector Pierna, Tobillo y Pie, Servicio de Ortopedia y Traumatología, Hospital Universitario Austral, Buenos Aires, Argentina \\ ** Departamento de Cirugía Ortopédica y Traumatológica, Clínica Universidad de Navarra, Navarra, España
}

\section{RESUMEN}

Introducción: Las técnicas para corregir las deformidades del hallux incluyen osteotomías metatarsianas y falángicas. Las osteotomías sobre la falange proximal corrigen el DASA y el ángulo interfalángico. Sin embargo, no se han publicado las indicaciones para la osteotomía de la falange distal. El objetivo de este artículo es comunicar la técnica y las indicaciones de la osteotomía percutánea de la falange distal del hallux, y evaluar los resultados de una serie de casos. Materiales y Métodos: Se analizaron 14 pies en los que se realizó una osteotomía de la falange distal del hallux para corregir una deformidad. Se midieron el DASA, la oblicuidad interfalángica y el ángulo falange distal-interfalángico en las radiografías. La técnica quirúrgica fue percutánea con control fluoroscópico. Los resultados se evaluaron mediante las escalas analógica visual de dolor y AOFAS. Seguimiento medio: 52 meses. Resultados: 13 pies de mujeres y un pie de hombre. Edad promedio: 58 años. Los resultados clínico y estético fueron excelentes, con alivio del dolor. Mejoría de la escala AOFAS: promedio 37 puntos. Análisis comparativo de ángulos preoperatorios y posoperatorios: DASA $(p=0,01)$, excepto cuando se aisló de la muestra a los pacientes con osteotomía tipo Akin $(p=0,33)$; ángulos F2-IF y F2-MTF ( $p<0,00001)$. Se registraron las complicaciones. Conclusiones: En la deformidad en valgo de la falange distal del hallux sintomática, se debe considerar una osteotomía correctora sola o asociada a osteotomía de la falange proximal. La osteotomía percutánea de la falange distal es un método eficaz, seguro y rápido.

Palabras clave: Osteotomía; falange; cirugía percutánea; hallux valgus; deformidad de hallux; osteotomía mínimamente invasiva. Nivel de Evidencia: IV

\section{Percutaneous Osteotomy of the Distal Phalanx of the Hallux. Indications and Technique}

\begin{abstract}
Introduction: There are many techniques to correct the hallux deformity. Most of them include metatarsal and/or phalanx osteotomies. The Akin osteotomy of the proximal phalanx is used to correct the distal articular set angle (DASA), or the interphalangeal angle. However, indications for the distal phalanx osteotomy remain unpublished. The aim of this study is to communicate the technique indications for percutaneous osteotomy of the distal phalanx of the hallux, and to evaluate the results of a case series. Materials and Methods: We reported 14 cases in which distal phalangeal osteotomy was performed. Radiographic measurements were performed on dorsal-plantar view of the foot, to analyze distal articular set angle (DASA), interphalangeal obliquity, and F2-IP angle. The surgical technique was performed by minimally incision surgery. The clinical and functional results were evaluated by the visual analog scale for pain and the AOFAS score. The average follow-up was 52 months. Results: The clinical outcomes for all the patients was excellent, pain was relieved and deformities corrected. Pre- and post-operative comparative angles: DASA (p: 0.01 ), except when isolated from the sample for Akin-type osteotomy ( $p: 0.33$ ). Angle F2-IP and angle F2-MTP ( $p:<0.00001)$. The patients where highly satisfied with both the aesthetic and functional results. Complications were registered. Conclusion: In the symptomatic hallux distal phalanx deformity a corrective distal phalanx osteotomy should be considered alone, or associated with the osteotomy of the proximal phalanx. Percutaneous distal phalanx osteotomy is an effective, safe, and fast procedure.
\end{abstract}

Key words: Phalangeal osteotomy; percutaneous surgery; hallux valgus; hallux deformity; MIS osteotomy.

Level of Evidence: IV

Recibido el 11-4-2020. Aceptado luego de la evaluación el 20-9-2020 • Dr. JUAN MANUEL YAÑEZ ARAUZ • juanyanezarauz@gmail.com (ID https://orcid.org/0000-0001-5739-3130 Cómo citar este artículo: Yañez Arauz JM, Yañez Arauz JM, Montiel Terrón V, Machado V, Alfonso M, Villas-Tomé C. Osteotomía percutánea de la falange distal del hallux. Indicaciones y técnica. Rev Asoc Argent Ortop Traumatol 2021;86(2):139-150. https://doi.org/10.15417/issn. 1852-7434.2021.86.2.1097 


\section{INTRODUCCIÓN}

Dentro de las patologías ortopédicas del primer dedo del pie, el hallux valgus es el principal motivo de consulta. Su etiología es multifactorial. ${ }^{1}$ Myerson ${ }^{2}$ sugiere una prevalencia de hallux valgus del 2-4\% de la población. La deformidad y sus manifestaciones clínicas aparecen entre la tercera y quinta décadas de la vida en el $65 \%$ de las personas y es mucho más frecuente en las mujeres. ${ }^{3,4}$

Se acepta que los calzados puntiagudos y con tacones altos contribuyen a su desarrollo, cambiando la configuración osteo-músculo-tendinosa estática y dinámica de los pies. ${ }^{5-10}$

El hallux valgus puede clasificarse de acuerdo con el sitio de deformidad del dedo (ubicación anatómica) en hallux valgus metatarsofalángico (MTF) en la mayoría de los casos o interfalángico (IF). En 1935, Daw ${ }^{11}$ define el término hallux valgus IF para describir una desviación lateral del dedo gordo del pie, a expensas de la articulación IF. Este autor menciona una desviación lateral de la falange distal $>10^{\circ}$, que puede ser independiente o asociarse a un hallux valgus MTF.

Sorto y cols. ${ }^{12}$ definieron los conceptos de "oblicuidad" o ángulo de valgo (AF1) como la relación de la diáfisis F1 con su superficie articular distal, y la "asimetría" o ángulo F2, como el ángulo formado por la perpendicular a la superficie de la articulación IF y el eje diafisario de la falange distal (Figura 1). Además, argumentan que las alteraciones del crecimiento de los cóndilos de las falanges, o de la base de la falange distal, producen deformidades a nivel IF.

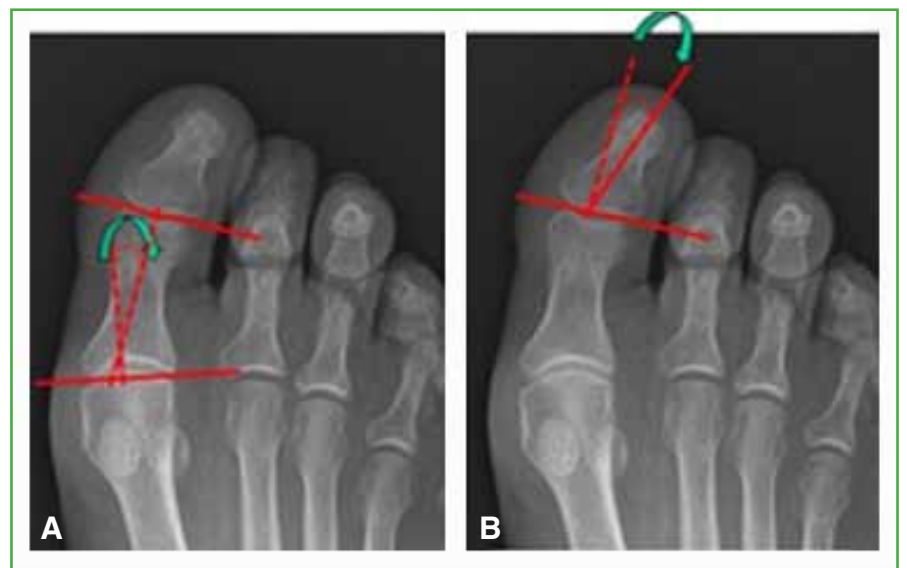

Figura 1. Medición de ángulos en las radiografías anteroposteriores con carga de peso. A. DASA. B. Ángulo F2.

En la práctica médica, las deformidades dolorosas del pie correspondientes al primer rayo, en general, son de resolución quirúrgica. El objetivo de la cirugía es corregir las deformidades óseas mediante osteotomías para obtener una correcta alineación biomecánica y una buena funcionalidad, y de esta manera, lograr un pie indoloro, plantígrado y con buen eje del primer rayo.

En mayoría de los casos, se recurre a las osteotomías para tratar el hallux valgus. En 1925, Akin ${ }^{13}$ describió un procedimiento para el hallux valgus que incluía la resección de la exostosis medial del primer metatarsiano, una osteotomía de resección en cuña de base medial en la base de la falange proximal del hallux y la liberación lateral del tendón abductor. Esta osteotomía falángica se popularizó como osteotomía de Akin, y fue descrita en la base de la falange proximal del hallux para la corrección del DASA (distal articular set angle) (Figura 2). Una variación de la osteotomía de Akin consiste en realizarla a nivel distal para corregir el hallux valgus IF (Figura 3). Se lleva a cabo en forma percutánea con técnica mínimamente invasiva.

Asimismo, se producen deformidades distales del hallux dolorosas como consecuencia de otras enfermedades, como diabetes, artritis inflamatorias, que no se asocian a hallux valgus. 


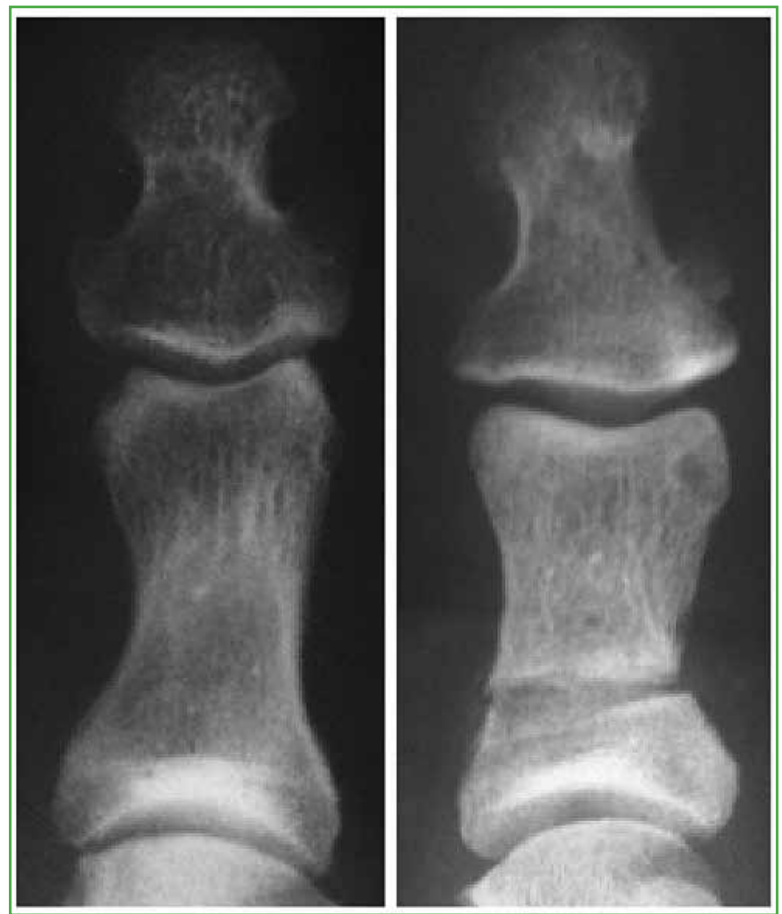

Figura 2. Osteotomía de Akin: corrige el DASA.

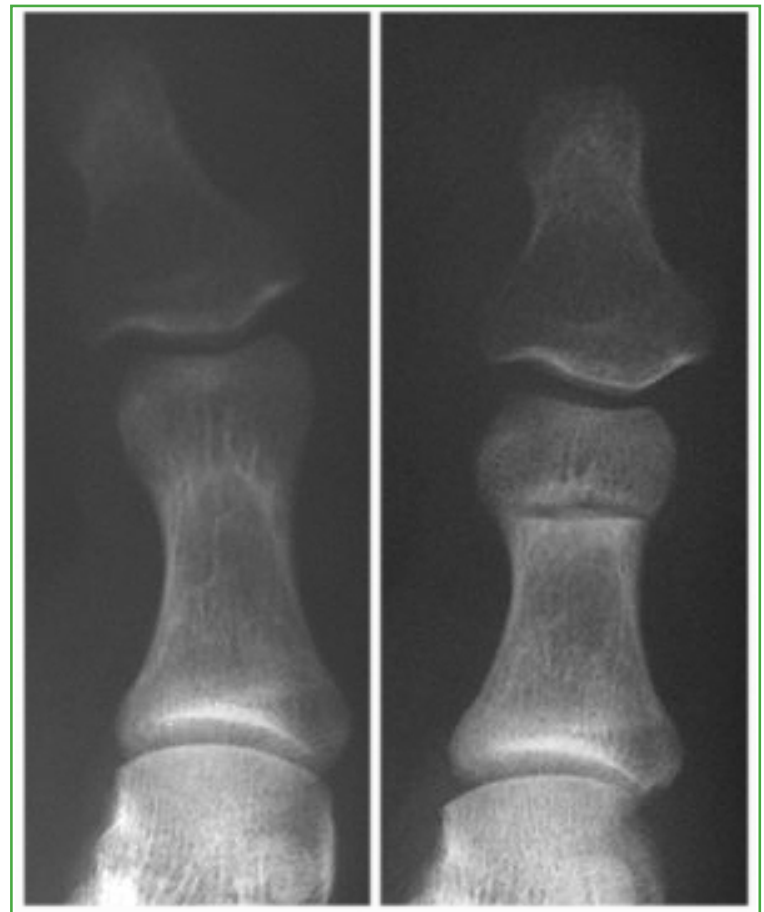

Figura 3. Osteotomía tipo Akin distal: corrige el ángulo interfalángico.

Teniendo en cuenta estos conceptos, el tratamiento de las deformidades del hallux (ya sea el hallux valgus con valgo IF o la deformidad distal única) debería realizarse en el sitio principal de la deformidad ósea, es decir, que la osteotomía podría efectuarse a nivel del metatarsiano, de la falange proximal o de la distal (de acuerdo con el centro de rotación del ángulo [CORA] de la deformidad); o bien mediante una combinación de ella, según el caso.

No hemos encontrado publicaciones que describan un algoritmo de tratamiento de la deformidad del hallux, mediante correcciones de desviaciones de la falange distal del hallux en forma percutánea.

El objetivo de este artículo es comunicar la técnica de la osteotomía de la falange distal del hallux por vía percutánea y sus indicaciones, y evaluar los resultados en una serie de casos.

\section{MATERIALES Y MÉTODOS}

Se trata de un estudio de serie de casos en el que se realizó una osteotomía de la falange distal del hallux para corregir una deformidad, asociada o no a cirugía de hallux valgus. Se analizaron 14 pies de 13 pacientes sometidos a una osteotomía en la base de la falange distal del hallux. En todos los casos, la osteotomía fue realizada por vía percutánea, de cuña sustractiva medial incompleta, sin necesidad de osteosíntesis, ya que, mediante osteoclasia, fue autoestable.

En cuanto a la enfermedad y deformidad previas: en cinco casos, se realizó esta osteotomía como parte de la cirugía de hallux valgus; cinco casos eran digitus valgus a expensas de una deformidad de la falange distal (uno bilateral) con dolor e incapacidad para calzarse; dos casos eran deformidades distales en pacientes diabéticos; un paciente presentaba una enfermedad inflamatoria crónica deformante y dolorosa; y el caso restante, una artrodesis MTF del hallux previa, con deformidad en valgo dolorosa de la falange distal.

El análisis clínico se realizó mediante la escala AOFAS del hallux y la escala analógica visual para dolor. ${ }^{14,15} \mathrm{El}$ seguimiento promedio fue de 52 meses (rango 39-92). 


\section{Análisis radiográfico}

Las mediciones se realizaron en radiografías de incidencia anteroposterior del pie, con el paciente parado sobre sus dos pies, soportando su peso corporal. Se registraron y analizaron los siguientes ángulos antes de la cirugía y en el posoperatorio a largo plazo (Figura 4):

1. DASA, trazando una tangente a la carilla articular proximal de la falange. De allí, se traza la perpendicular a la tangente, y se mide el ángulo que forma esta perpendicular con la línea del eje diafisario de la falange proximal. Los valores normales son de $0^{\circ}$ a $8^{\circ}$.

2. La oblicuidad de la articulación interfalángica $(\mathrm{OIF})^{12}$ que se mide tomando el ángulo donde se cruzan las perpendiculares a las líneas tangentes de ambas carillas articulares (proximal y distal de la primera falange).

3. La oblicuidad F2-interfalángico (F2-IF) ${ }^{12}$ que se mide por el ángulo formado entre el eje de la falange distal y la perpendicular a la tangente a los cóndilos de la falange proximal.

4. El ángulo falange distal-articulación MTF (F2-MTF): se toma la resultante del entrecruzamiento de la tangente a la base de la primera falange y el eje longitudinal de la falange distal.

Se consideró que las alteraciones angulares que se produjeron después de los 38 meses obedecían a otras causas y no a la técnica quirúrgica en sí (p. ej., osteoartrosis, inestabilidad cuneo-metatarsiana, desarrollo de hallux valgus, etc.).

Todas las imágenes fueron digitales, y las mediciones se realizaron mediante el programa computarizado (MB Rouler® versión 4.0)
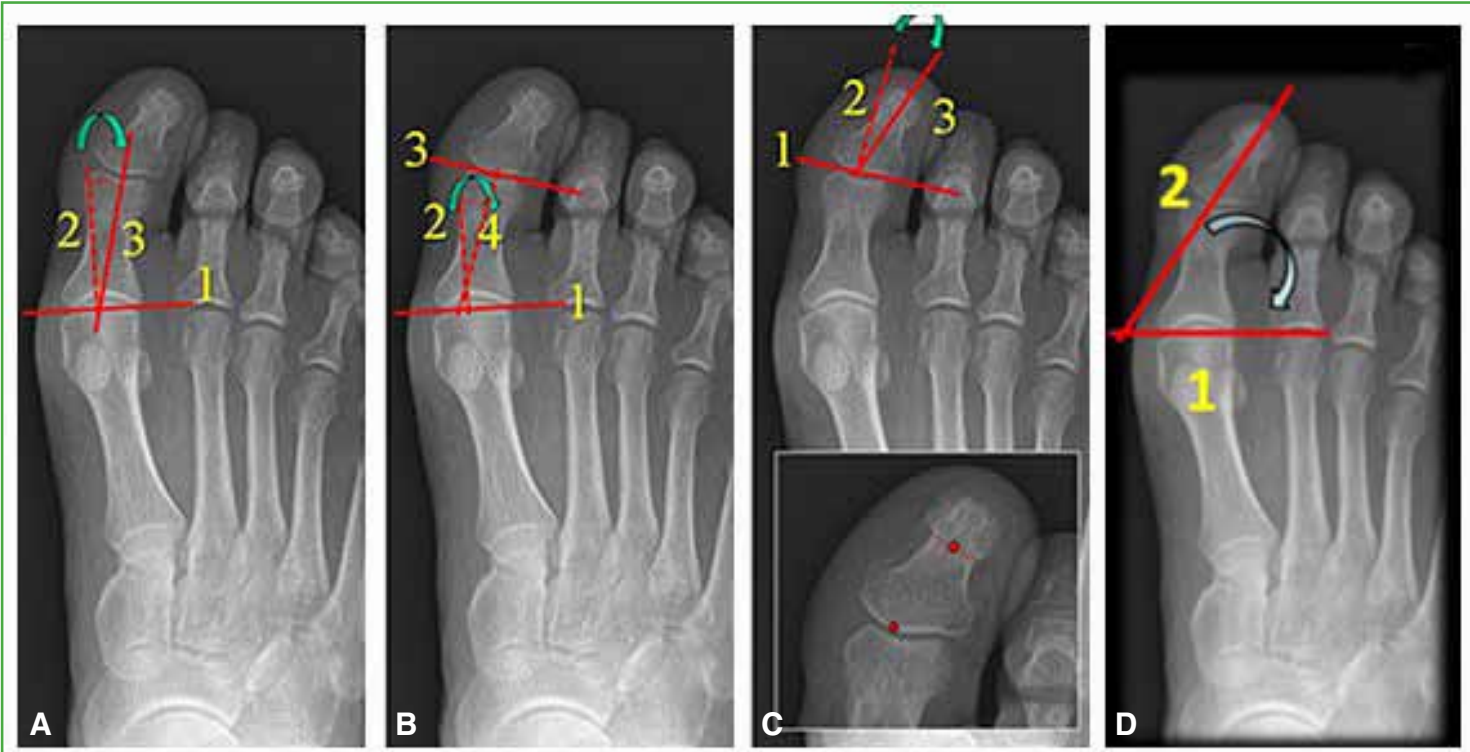

Figura 4. Mediciones radiográficas. A. Medición del DASA. B. Oblicuidad interfalángica. C. Oblicuidad F2interfalángica. D. Ángulo metatarsofalángico-falange distal.

\section{Análisis estadístico}

Para el análisis de las diferencias en la distribución de una variable entre el preoperatorio y el posoperatorio, se utilizó la prueba t para muestras emparejadas, y se consideró estadísticamente significativo un valor p <0,05.

Se describen dos casos a modo de ejemplo: 


\section{CASO 1}

Mujer de 67 años que consultó por desviación en valgo de la falange distal del primer dedo del pie izquierdo y dolor en la articulación IF asociado a limitación a la extensión del segundo dedo que provocó un dedo en garra.

Las radiografías anteroposteriores preoperatorias del pie izquierdo mostraron un DASA de $3^{\circ}$, una OIF de $5^{\circ}$, un ángulo F2-IF de $3,5^{\circ}$ y un ángulo F2-MTF de $78^{\circ}$. Después de considerar las posibles opciones quirúrgicas y las complicaciones, se realizó una corrección quirúrgica que incluyó una osteotomía percutánea de la falange distal del hallux varizante, asociada a una artrodesis IF del segundo dedo del pie izquierdo. La cirugía y el período posoperatorio se realizaron de acuerdo con lo explicado más adelante, en la descripción de la técnica quirúrgica (Figura 5). Los valores a los 9 meses de la cirugía fueron: DASA $1,5^{\circ}$, OIF $3^{\circ}$, ángulo F2-IF - $4^{\circ}$, ángulo F2-MTF $112^{\circ}$. La paciente tuvo una buena evolución, el dolor se alivió, y estaba satisfecha con los resultados.

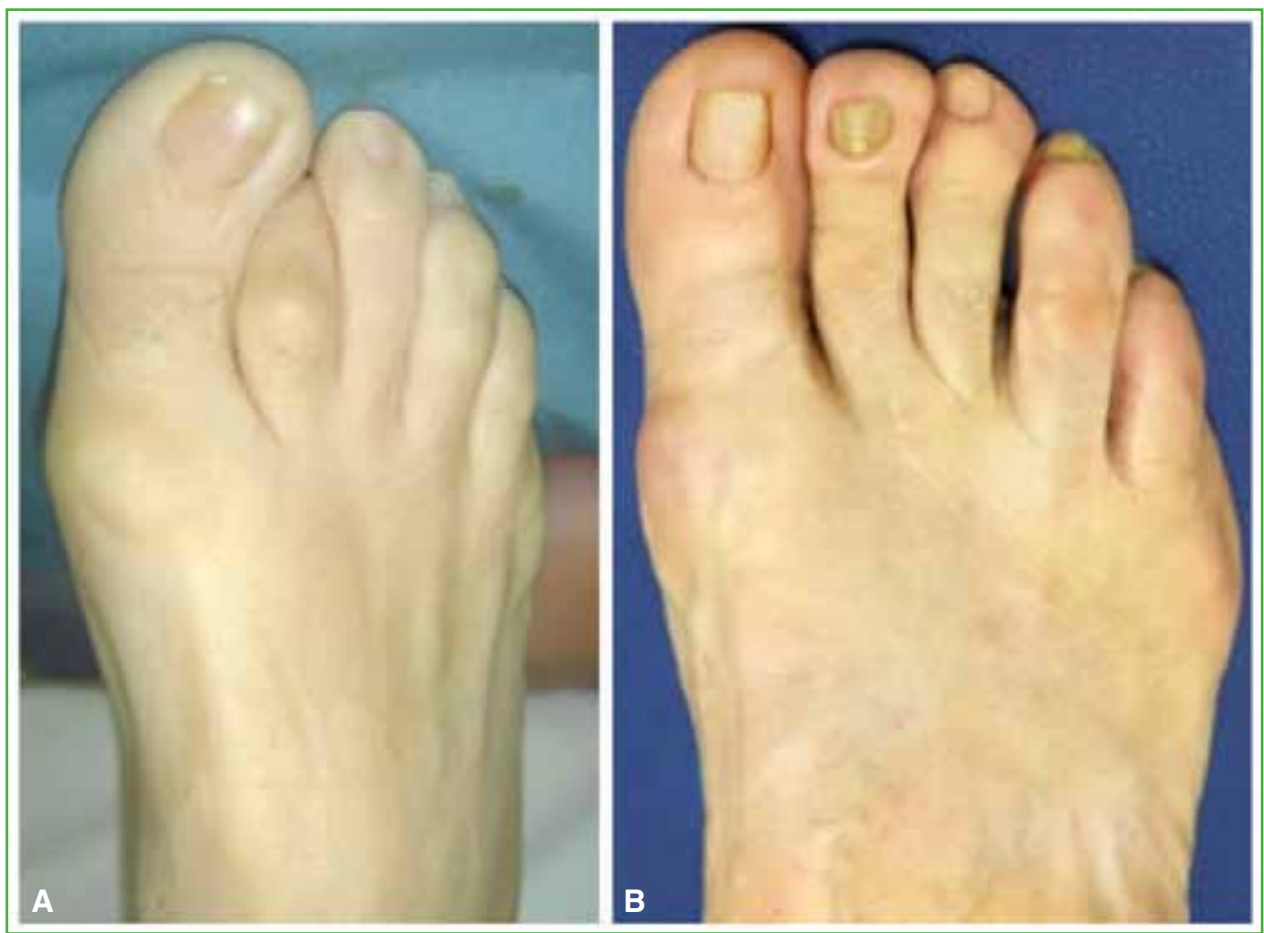

Figura 5. Caso 1. Deformidad distal de hallux con dolor articular y segundo dedo en martillo doloroso. A. Imagen preoperatoria. B. Imagen a los 9 meses de la cirugía.

\section{CASO 2}

Mujer de 42 años con una deformidad en valgo de la falange distal del primer dedo del pie derecho, con dolor ungueal y que interrumpe al segundo dedo causando un dedo en martillo doloroso con dificultad para usar el calzado habitual (Figura 6A), ya que le quita el espacio de localización del segundo dedo. Las radiografías del pie derecho mostraron un DASA de $5^{\circ}$, una OIF de $12^{\circ}$, un ángulo F2-IF de $10^{\circ}$ y un ángulo F2-MTF de $60^{\circ}$ (Figura 6B) antes de la cirugía. Se llevó a cabo la corrección quirúrgica mediante una osteotomía de cierre percutánea de la base de la tercera falange del hallux, asociada a tratamiento del segundo dedo en martillo, mediante técnica mínimamente invasiva, con osteotomía de la base de la primera falange y tenotomía del extensor del segundo dedo (Figura 6C y D). Los valores posoperatorios fueron: DASA $6^{\circ}$, OIF $4^{\circ}$, ángulo F2-IF $6^{\circ}$ y ángulo F2-MTF $88^{\circ}$. La evolución fue satisfactoria con alivio del dolor y la posibilidad de usar el calzado habitual. 


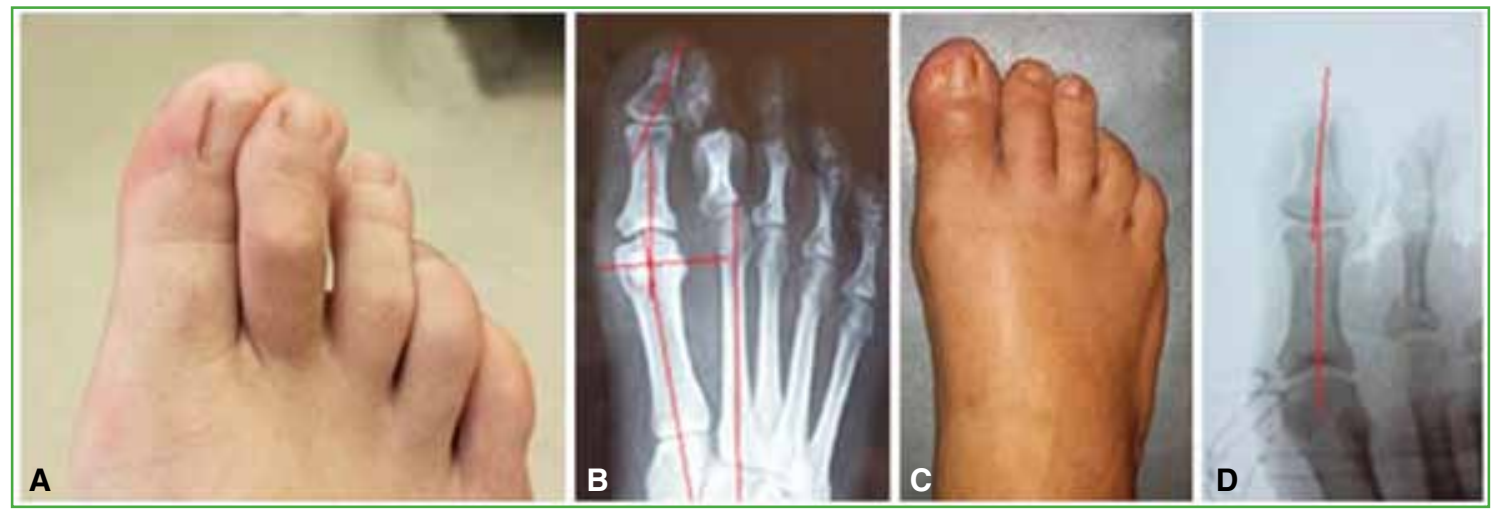

Figura 6. Caso 2. Deformidad distal de hallux con dolor y deformidad ungueal, y dolor en el segundo dedo.

A. Imagen preoperatoria. B. Radiografía preoperatoria. C. Imagen a los 2 meses de la cirugía.

D. Radiografía a las 6 semanas de la cirugía.

\section{Técnica quirúrgica percutánea de osteotomía de la falange distal del hallux}

Se describen los pasos principales: se coloca al paciente en decúbito dorsal con anestesia troncular en el hallux por operar. Se realiza un portal medial en la base de la falange distal $5 \mathrm{~mm}$ distal a la articulación, con bisturí Beaver 64, bajo control fluoroscópico, hasta llegar al hueso. Tener como referencia el comienzo de la matriz ungueal y proceder con cuidado para no lesionarla, ya que está inmediatamente distal al sitio de la osteotomía.

Luego, con una fresa Shannon corta a bajas revoluciones, se realiza una osteotomía incompleta de base medial paralela a la superficie articular para no lesionar la matriz que, según el ángulo de deformidad por corregir, su base será mayor o menor. Se controla con fluoroscopia. Se mantiene intacta la pared lateral de la falange distal y su periostio. Esto permite que la osteotomía sea autoestable (Figura 7).

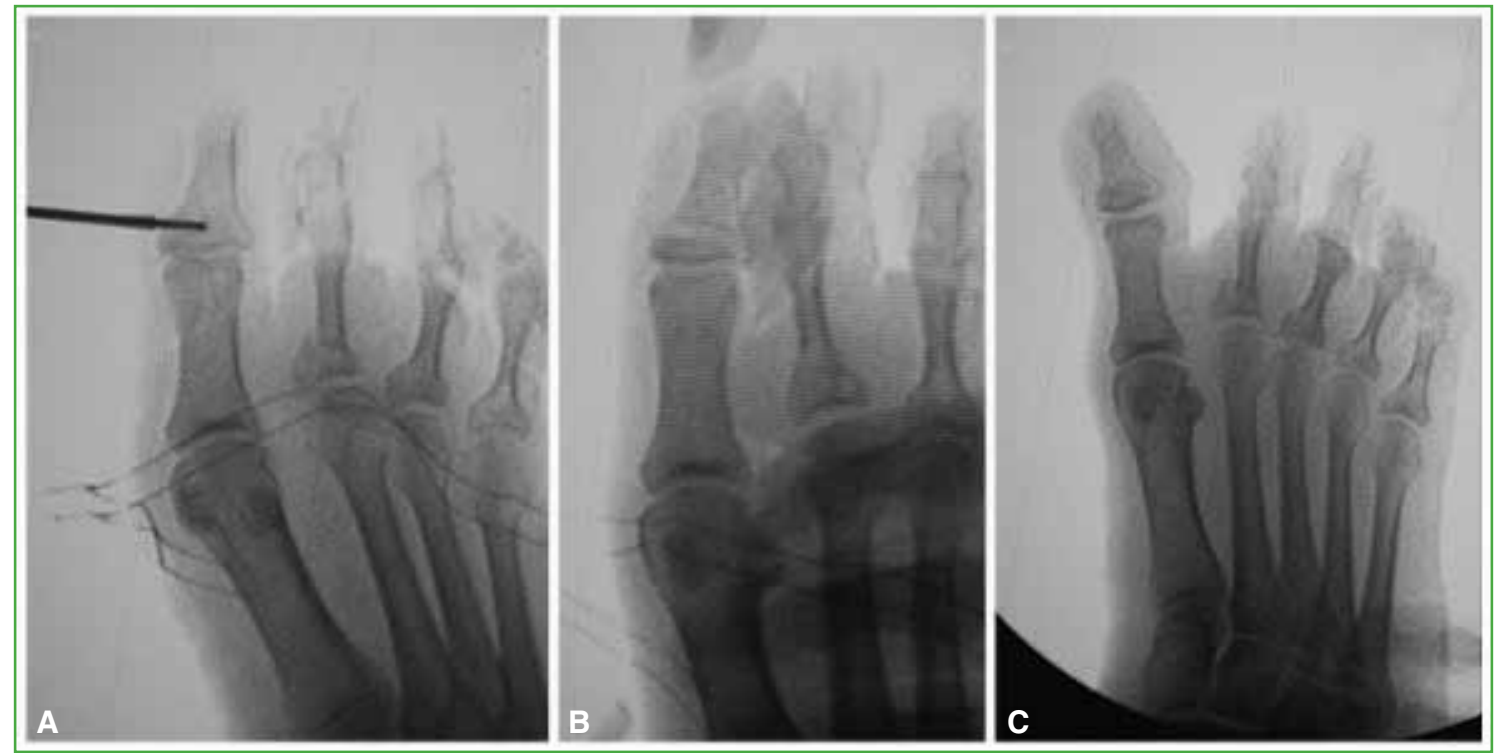

Figura 7. A. Técnica de osteotomía incompleta de base de falange distal en forma percutánea con fresa Shannon corta. B. Osteotomía de base medial. C. Osteoclasia con corrección de la deformidad. 
Por último, se procede con la osteoclasia y la corrección de la deformidad. Control con fluoroscopia. Cierre con un punto de nailon 4.0 (Figura 8). Vendaje corrector durante tres semanas, manteniendo el objetivo logrado, para conseguir la consolidación en la posición deseada. Calzado de posoperatorio con apoyo inmediato. Al mes de la cirugía, se permite el uso progresivo del calzado normal, según la tolerancia.

Nota: En caso de inestabilidad de la osteotomía por sección completa ósea, se puede estabilizar transitoriamente con una aguja de Kirschner por tres semanas para evitar desviaciones inadecuadas. Esto es poco probable con la cirugía mínimamente invasiva.

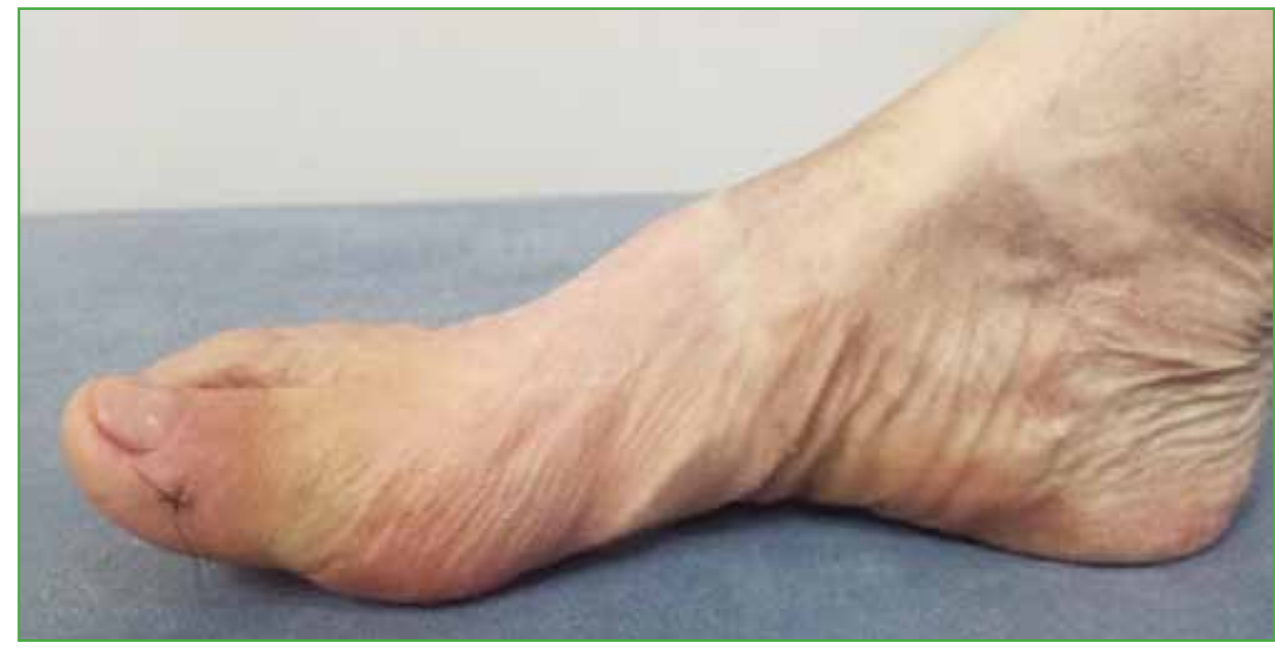

Figura 8. Imagen del posoperatorio inmediato.

\section{RESULTADOS}

Trece de los 14 pies operados correspondían a mujeres (un caso fue bilateral) y uno, a un hombre. La edad promedio era de 58 años (rango 40-74). Los resultados clínico y estético fueron excelentes en todos los pacientes, con alivio del dolor y buen resultado cosmético subjetivo. Se corrigió la deformidad previa de la falange distal del hallux de todos los pacientes.

En cuanto a la escala AOFAS, se observó una mejoría promedio de 37 puntos, con una media de 50,7 (rango 3565 ) antes de la cirugía y de 87,5 (rango 75-100) en el posoperatorio (Tabla 1).

Con respecto al dolor, se registró un promedio de dolor de 4,64 (rango 2-7) antes de la intervención y de 1 (rango $0-2)$ en el posoperatorio $(\mathrm{p}<0,0001)$ (Tabla 1$)$.

Tabla 1. Resultados de la escala analógica visual y la escala AOFAS

\begin{tabular}{|c|c|c|c|c|c|}
\hline & \multicolumn{4}{|c|}{ Puntaje AOFAS } & \multicolumn{2}{c|}{ Valores de referencia } \\
\hline & Preoperatorio & Posoperatorio & Mejoría & $\mathrm{p}$ & De 0 a 100 \\
\hline Promedio & 50,7 & 87,5 & 36,8 & $\mathrm{p}<0,0001$ & $0=$ resultado malo \\
\hline
\end{tabular}

\begin{tabular}{|c|c|c|c|c|c|}
\hline & \multicolumn{4}{|c|}{ Escala analógica visual (de 0 a 10) } & \multicolumn{2}{c|}{ Valor de referencia } \\
\hline & Preoperatorio & Posoperatorio & Mejoría & $\mathrm{p}$ & $0=$ sin dolor \\
\hline Promedio & 4,64 & 1 & 3,64 & $\mathrm{p}<0,0001$ & $10=$ dolor intolerable \\
\hline
\end{tabular}


En la Tabla 2, se muestran las mediciones radiográficas de los ángulos en el preoperatorio y en el posoperatorio alejado, en forma comparativa.

En cinco casos, se agregó una osteotomía percutánea tipo Akin de la primera falange (pacientes 3, 8, 9, 11 y 12) y se observó una mejoría del DASA en las mediciones posoperatorias, por lo que se llega a la conclusión de que la osteotomía descrita por Akin es una buena técnica correctora del DASA.

Tabla 2. Mediciones radiográficas de los valores angulares antes de la cirugía y en el posoperatorio

\begin{tabular}{|c|c|c|c|c|c|c|c|c|c|}
\hline \multirow{2}{*}{ Caso } & \multicolumn{8}{|c|}{ Mediciones preoperatorias y posoperatorias de los ángulos medidos } & \multirow[t]{2}{*}{ Observaciones } \\
\hline & \multicolumn{2}{|c|}{ DASA } & \multicolumn{2}{|c|}{ OIF } & \multicolumn{2}{|c|}{ F2-IF } & \multicolumn{2}{|c|}{ F2-MTF } & \\
\hline \multirow{2}{*}{$\begin{array}{l}\text { Valores de } \\
\text { referencia }\end{array}$} & \multicolumn{2}{|c|}{ De $0^{\circ}$ a $8^{\circ}$} & \multicolumn{2}{|c|}{ De $0^{\circ}$ a $10^{\circ}$} & \multicolumn{2}{|c|}{$\operatorname{De} 0^{\circ}$ a $5^{\circ}$} & \multicolumn{2}{|c|}{ De $87^{\circ}$ a $95^{\circ}$} & \\
\hline & Preop. & Posop. & Preop. & Posop. & Preop. & Posop. & Preop. & Posop. & \\
\hline 1 & 3 & 1,5 & 5 & 3 & 3,5 & -4 & 78 & 102 & \\
\hline 2 & 4 & 4 & 11 & 15 & 3 & 1,5 & 71 & 85 & \\
\hline 3 & 12 & 1 & 2 & 6 & 16 & 2 & 55 & 85 & $\begin{array}{c}\text { Se agregó osteotomía } \\
\text { de F1 }\end{array}$ \\
\hline 4 & 7 & 7 & 14 & 13 & 12 & 4 & 67 & 86 & \\
\hline 5 & 5 & 6 & 12 & 4 & 10 & 6 & 60 & 88 & \\
\hline 6 & 15 & 9 & 5 & 5 & 15 & 7 & 70 & 91 & \\
\hline 7 & 2 & 2 & 3 & 3 & 17 & 4 & 72 & 86 & \\
\hline 8 & 16 & 3 & 12 & 8 & 5 & 2 & 86 & 95 & $\begin{array}{c}\text { Se agregó osteotomía } \\
\text { de F1 }\end{array}$ \\
\hline 9 & 10 & 1 & 6 & 0 & 12 & 3 & 73 & 88 & $\begin{array}{c}\text { Se agregó osteotomía } \\
\text { de F1 }\end{array}$ \\
\hline 10 & 3 & 3 & 18 & 18 & 16 & -2 & 67 & 90 & \\
\hline 11 & 14 & 7 & 17 & 12 & 4 & 0 & 82 & 90 & $\begin{array}{c}\text { Se agregó osteotomía } \\
\text { de F1 }\end{array}$ \\
\hline 12 & 17 & 4 & 12 & 5 & 16 & 5 & 65 & 85 & $\begin{array}{c}\text { Se agregó osteotomía } \\
\text { de F1 }\end{array}$ \\
\hline 13 & 9 & 9 & 5 & 5 & 15 & 2 & 68 & 88 & \\
\hline 14 & 8 & 8 & 8 & 10 & 19 & 3 & 63 & 86 & \\
\hline
\end{tabular}

DASA = distal articular set angle, $\mathrm{OIF}=$ oblicuidad de la articulación interfalángica, F2-IF = ángulo oblicuidad F2-interfalángico, F2-MTF = ángulo falange distal-articulación metatarsofalángica.

El análisis comparativo de los valores angulares entre el preoperatorio y el posoperatorio de la osteotomía de la falange distal, arrojó lo siguiente:

a) el DASA mostró una diferencia significativa $(p=0,01)$ cuando se tomaron todos los valores juntos. Si se aísla a quienes se les realizó una osteotomía tipo Akin de la falange proximal, la diferencia no fue estadísticamente significativa ( $\mathrm{p}=0,33$ ); esto significa que la osteotomía de la falange distal pura no altera el DASA.

b) El ángulo OIF no tuvo una diferencia significativa $(\mathrm{p}=0,13)$.

c) La medición del ángulo F2-IF presentó una diferencia significativa ( $\mathrm{p}=0,00001)$ (Tabla 3). 
d) El análisis del ángulo F2-MTF también arrojó una diferencia estadísticamente significativa $(\mathrm{p}=0,000001)$ (Tabla 3).

e) Estos últimos dos ítems demuestran el poder de corrección de la osteotomía de F2 (o falange distal) en los ángulos F2-IF y F2-MTF.

No hubo complicaciones durante la cirugía. En el posoperatorio, solo un paciente tenía alteración del crecimiento ungueal asintomática.

Tabla 3. Promedio de ángulos preoperatorios y posoperatorios, y significancia estadística de corrección

\begin{tabular}{|c|c|c|c|c|c|}
\hline \multicolumn{2}{|c|}{ Medición promedio ángulo F2-IF $\left(0^{\circ}-5^{\circ}\right)$} & \multicolumn{3}{c|}{ Medición promedio ángulo F2-MTF $\left(8^{\circ}-95^{\circ}\right)$} \\
\hline Preoperatorio & Posoperatorio & $p$ & Preoperatorio & Posoperatorio & $p$ \\
\hline $11,67^{\circ}$ & $2,36^{\circ}$ & $<0,0001$ & $69,8^{\circ}$ & $88,9^{\circ}$ & $<0,0001$ \\
\hline
\end{tabular}

F2-IF = ángulo oblicuidad F2-interfalángico, F2-MTF = ángulo falange distal-articulación metatarsofalángica.

\section{DISCUSIÓN}

Se han propuesto diferentes algoritmos de tratamiento para la deformidad del hallux valgus y del valgus IF mediante técnicas quirúrgicas que incluyen alineación ósea en los diferentes planos, asociada a otros pasos quirúrgicos, como la resección de la prominencia medial de la cabeza metatarsiana y acciones sobre las partes blandas. ${ }^{16}$

A pesar de su importancia, no hay datos claros sobre la prevalencia de hallux valgus IF. Tampoco hay consenso claro sobre el ángulo IF normal (AIF) o las causas que pueden conducir a su deformidad. Barnett ${ }^{17}$ sugirió un ángulo promedio de $5^{\circ}$ como normal y Bryant, ${ }^{18}$ un promedio de $5^{\circ}$ en pacientes con hallux valgus y de $15^{\circ}$ con hallux rigidus.

Sorto y cols. ${ }^{12}$ introdujeron la posible influencia de la estabilidad de la articulación MTF e IF, y una relación inversa entre la presencia de hallux valgus MTF y hallux valgus IF.

Coughlin y Shurnas ${ }^{19}$ estudiaron 127 pies con hallux rigidus y observaron un ángulo IF aumentado (promedio $18^{\circ}$ ), y concluyeron en que la rigidez por osteoartritis MTF fue la causa del incremento angular IF y la inestabilidad.

Coincidiendo con estos autores, Sorto y cols. ${ }^{12,20}$ mencionan que la rigidez de la articulación MTF es un factor desencadenante que determina la inestabilidad de la articulación IF. Esto explica la asociación entre hallux valgus IF y valgo de la falange distal, en pacientes con artrodesis MTF.

Fitzgerald $^{21}$ estudió a largo plazo una serie de 69 pacientes con artrodesis MTF, y halló un aumento del ángulo IF con el desarrollo de cambios artríticos en esa articulación, después de la operación. En nuestra serie, tuvimos un paciente con artrodesis MTF y desviación secundaria de la falange distal en valgo dolorosa, tratada con la técnica descrita.

Barnett $^{22}$ estableció que la asimetría y la oblicuidad son los factores que causan la deformidad de hallux valgus IF.

Shimizu y cols. ${ }^{23}$ publican un caso de desarrollo de hallux valgus IF luego de una lesión de la epífisis por trauma osteocondral articular, en un paciente joven.

Por todo lo expuesto es que existen múltiples procedimientos para tratar las deformidades del hallux.

En ocasiones, luego de haber elegido una correcta técnica quirúrgica, se observan hipocorrecciones o hipercorrecciones, e incluso recidivas de la deformidad. Otras veces, el problema surge de una evaluación inadecuada de la deformidad y la elección de una técnica incorrecta. Es aquí donde la falange distal del hallux, poco valorada dentro del primer radio, puede causar insatisfacción en algunos casos debido a la deformidad residual, que consiste en una desviación de la uña y la falange distal hacia el lado peroneo después de la cirugía, y el dolor y la deformidad consiguientes en el segundo dedo del pie.

En la bibliografía, solo encontramos la descripción de una osteotomía a cielo abierto en la falange distal de apertura lateral, mediante abordaje externo, publicada por Carnevale ${ }^{24}$ y que había sido descrita por los doctores José María del Sel y Scaramuzza en el Primer Congreso de COT, Mar del Plata, Argentina, en 1956. 
Considerando la deformidad articular IF, la deformidad intrínseca de la falange proximal, y la deformidad de la falange distal, creemos que la solución del tratamiento quirúrgico podría estar dirigida a la falange proximal, a la distal o a ambas en conjunto.

Hay otros casos en los que la limitación funcional se produce debido a una deformidad dolorosa del segundo dedo del pie causada por la desviación lateral de la falange distal del hallux, sin alteración de la articulación MTF. Este tipo de desviación distal no suele alterar la movilidad del dedo del pie, pero puede causar problemas mecánicos debido al frotamiento con el segundo dedo, y la "falta de espacio" con el uso de calzado inapropiado, lo que causará una mayor deformidad recíprocamente. Dado que todo el lecho ungueal parece seguir la dirección de la falange distal, ${ }^{25}$ estas deformidades pueden causar trastornos tróficos, como la onicocriptosis.

El aumento del crecimiento del lado tibial de la falange distal observado en algunos casos determina que sea más largo en comparación con el borde peroneo, y podría ser la causa de la deformidad en valgo distal, o simplemente provocar el crecimiento de la exostosis ósea ${ }^{26}$ como resultado de la inhibición de la fisis lateral o la estimulación relativa mayor de la epífisis medial.

Barouk y cols. ${ }^{27}$ mencionan que las deformidades en valgo de la articulación IF se tratan con técnicas que involucran la falange proximal, como la osteotomía descrita por Akin. ${ }^{18}$ Pero esta sería insuficiente para corregir la asimetría que está condicionada a la deformidad de la falange distal.

Vander Griend ${ }^{28}$ evaluó 33 pies operados mediante osteotomía distal de la primera falange en pacientes con hallux valgus IF, y llegó a la conclusión de que se trata de una buena técnica para corregir este cuadro, que es segura, provoca pocas complicaciones, y logra buenos resultados clínicos y de satisfacción del paciente. Pero, en su estudio, no menciona los casos, aunque pocos, en los que la deformidad está en la falange distal del hallux. Por lo tanto, si queremos actuar sobre el centro de rotación del ángulo de la deformidad y corregir la deformidad distal y el valgo IF, deberíamos recurrir a una osteotomía en la falange distal, que es lo que se presenta en este estudio. Esto se debe a que la osteotomía en la falange proximal no corregirá la deformación distal, como lo describieron Sorto y cols. ${ }^{12}$

En 1957, Burry ${ }^{29}$ ideó un método conciso para cuantificar la cantidad de desviación de valgo en la articulación, a través de la relación angular entre las bisecciones longitudinales de las falanges distal y proximal del hallux (ángulo IF). Usando este método, Barnett evaluó 346 pies, ${ }^{17}$ y calculó una desviación promedio de valgo IF de $13^{\circ}$ a $13,58^{\circ}$. Sorto y cols. ${ }^{12}$ reforzaron este hallazgo, determinando que el ángulo promedio era de $13,48^{\circ}$ en 432 pies evaluados.

Un estudio cadavérico menciona como complicación de la osteotomía en la falange distal el posible daño del lecho ungueal y la lesión del extensor largo del hallux. ${ }^{30}$ Creemos que el conocimiento depurado de la anatomía normal y la realización delicada de la técnica quirúrgica reducirían al mínimo estas posibilidades de lesión. En nuestra serie, solo observamos un paciente con leves trastornos tróficos del crecimiento ungueal, totalmente asintomático. No hemos detectado lesión del extensor del hallux.

Los autores creen que la deformidad de la falange distal no causaría problemas cuando se trata de ángulos pequeños entre las falanges, pero puede provocar insatisfacción en deformidades moderadas o severas, especialmente con ángulos IF o ángulo F2-IF $>20^{\circ}$.

No existe un consenso claro sobre cuál es el ángulo normal ideal de la falange distal; por lo tanto, surge la pregunta de si debemos buscar una compensación. Sin embargo, de acuerdo con los estudios mencionados, los ángulos $\mathrm{F} 2-\mathrm{IF}>20^{\circ}$ sintomáticos deberían ser tratados mediante una osteotomía de la falange distal.

Otro caso es la deformidad pura de la falange distal, con dolor y deformidad por compresión del segundo dedo, y eventual alteración dolorosa ungueal del hallux. En estas situaciones, se debería realizar una osteotomía correctora de la falange distal.

Finalmente, cabe mencionar los casos de hipocorrección IF luego de una cirugía de hallux valgus, con desviación lateral distal y dolor residual localizado en el área tibial de la articulación IF con queratosis local. En tales casos, la cirugía sobre la falange distal podría estar indicada.

Como fortaleza de este estudio se mencionan el análisis estadístico, su originalidad y lo inédito en la literatura. También el seguimiento superior a tres años en todos los casos. Como debilidades se mencionan la cantidad de casos presentados, pero hay que tener en cuenta que son deformidades sintomáticas poco frecuentes. 


\section{CONCLUSIONES}

En pacientes con una desviación en valgo $>20^{\circ}$ de la falange distal del hallux sintomática, se debe considerar una osteotomía percutánea correctora de dicha falange sola o asociada a una osteotomía de la falange proximal. Lo mismo se aplica a los casos de desviación distal con compromiso sintomático del segundo dedo.

A pesar de la muestra pequeña, creemos que la osteotomía percutánea de la falange distal del hallux permitiría lograr una buena corrección clínica y radiográfica en las deformidades distales del hallux. La osteotomía percutánea de la falange distal del hallux parece ser un método eficaz, seguro y de rápida ejecución.

Conflicto de intereses: Los autores no declaran conflictos de intereses.

ORCID de J. M. Yañez Arauz: https://orcid.org/0000-0003-3321-3726 ORCID de V. Montiel Terrón: https://orcid org/0000-0002-2140-427X ORCID de V. Machado: https://orcid.org/0000-0002-0162-9383
ORCID de M. Alfonso: https://orcid.org/0000-0001-5824-0857 ORCID de C. Villas-Tomé: https://orcid.org/0000-0002-7364-6967

\section{BIBLIOGRAFÍA}

1. Mann RA, Coughlin MJ. Hallux valgus-etiology, anatomy, treatment and surgical considerations. Clin Orthop Relat Res 1981;(157):31-41. PMID:7249460

2. Myerson MS. Hallux valgus. En: Myerson MS (ed.). Foot and ankle disorders. Philadelphia: Saunders; 2000.

3. Coughlin MJ, Thompson FM. The high price of high-fashion footwear. Instr Course Lect 1995;44:371-7. PMID: 7797875

4. Coughlin MJ. Hallux valgus in men: Effect of the distal metatarsal articular angle on hallux valgus correction. Foot Ankle Int 1997;18(8):463-70. https://doi.org/10.1177/107110079701800802

5. Haines RW. Shoe design and toe deformation. Lancet 1954; 267(6852):1308-11. https://doi.org/10.1016/s0140-6736(54)90712-6

6. Lelievre J. Shoes and our foot. III. The physiological shoe. Concours Med 1961;83:1081-4. PMID: 13760672

7. Frey C, Thompson F, Smith J. Update on women's footwear. Foot Ankle Int 1995;16(6):328-31. https://doi.org/10.1177/107110079501600603

8. Craigmile DA. Incidence, origin, and prevention of certain foot defects. Br Med J 1953;2(4839):749-52. https://doi.org/10.1136/bmj.2.4839.749

9. Coughlin MJ. Women's shoe wear and foot disorders. West J Med 1995;163(6):569-70. https://doi.org/10.1177/107110079501600603

10. Coughlin MJ. Roger A. Mann Award. Juvenile hallux valgus: etiology and treatment. Foot Ankle Int 1995;16(11): 682-97. https://doi.org/10.1177/107110079501601104

11. Daw SW. An unusual type of hallus valgus (two cases). BMJ 1935; 2:580-2.

12. Sorto Jr LA, Balding MG, Weil LS, Smith SD. Hallux abductus interphalangeus. Etiology, X-ray evaluation and treatment. 1975. J Am Podiatr Med Assoc 1992;82(2):85-97. https://doi.org/10.7547/87507315-82-2-85

13. Akin OF. The treatment of hallux valgus-a new operative procedure and its results. Medical Sentinel 1925;33:678.

14. Hayes MHS, Patterson DG. Experimental development of the graphic rating method. Psychological Bulletin 1921;18:98-99.

15. Zealley AK, Aitken RCB. A growing edge of measurement of feelings [Abridged]: Measurement of mood. Proc $R$ Soc Med 1969;62(10):993-6. PMID: 5346176

16. Mann R, Coughlin M. Hallux valgus y sus complicaciones. En: Cirugía del pie, $5^{\mathrm{a}}$ ed. Buenos Aires: Editorial Médica Panamericana; 1986.

17. Barnett CH. Valgus deviation of the distal phalanx of the great toe. J Anat 1962;96(Pt 2):171-7. PMID: 13865114

18. Bryant JA. A comparison of radiographic foot measurements taken in two different positions. J Am Podiatr Med Assoc 2001;91(5):234-9. https://doi.org/10.7547/87507315-91-5-234 
19. Coughlin MJ, Shurnas PS. Hallux rigidus: demographics, etiology, and radiographic assessment. Foot Ankle Int 2003;24(10):731-43. https://doi.org/10.1177/107110070302401002

20. Sorto LA, Balding MG, Weil LS, Smith SD. Hallux abductus interphalangeus: etiology, x-ray evaluation and treatment. J Am Podiatry Assoc 1976;66(6):384-96. https://doi.org/10.7547/87507315-66-6-384

21. Fitzgerald JA. A review of long-term results of arthrodesis of the first metatarso-phalangeal joint. J Bone Joint Surg Br 1969;51(3):488-93. PMID: 5820792

22. Barnett CH. The normal orientation of the human hallux and the effect of footwear. $J$ Anat 1962;96(Pt 4):489-94. PMID: 13969386

23. Shimizu A, Watanabe S, Kamada K, Tsuboi I, Yamamoto H. Hallux valgus interphalangeus following osteochondral fracture of the proximal phalanx: a case report. Foot Ankle Int 2005;26(11):994-6. https://doi.org/10.1177/107110070502601116

24. Carnevale V. Phalanx valga of the great toe ("phalanx valga hallucis") or clinodactyly of the great toe. Prensa Médica Argentina 1959;46:761-3.

25. Parrinello JF, Japour CJ, Dykyj D. Incurvated nail. Does the phalanx determine nail plate shape? J Am Podiatr Med Assoc 1995;85:696-8. https://doi.org/10.7547/87507315-85-11-696

26. Villas C, Del Rio J, Valenti A, Alfonso M. Symptomatic medial exostosis of the great toe distal phalanx: a complication due to over-correction following Akin osteotomy for hallux valgus repair. J Foot Ankle Surg 2009;48(1):47-51. https://doi.org/10.1053/j.jfas.2008.08.011

27. Barouk LS, Barouk P, Baudet B, Toullec E. The great toe proximal phalanx osteotomy: the final step of the bunionectomy. Foot Ankle Clin 2005;10(1):141-55. https://doi.org/10.1016/j.fcl.2004.09.006

28. Vander Griend R. Correction of hallux valgus interphalangeus with an osteotomy of the distal end of the proximal phalanx (distal Akin osteotomy). Foot Ankle Int 2017;38(2):153-8. https://doi.org/10.1177/1071100716670389

29. Burry HAS. The effect of shoes on hallux valgus: a significant study. Research Report 147: British Boot, Shoe and Allied Trades Research Association.

30. Valentí A, Montiel V, Alfonso M, Villas C. Feasibility of open and percutaneous corrective osteotomies of the second phalanx of the great toe. An approach on a cadaveric model. Foot (Edinb) 2019;38:39-42. https://doi.org/10.1016/j.foot.2018.12.001 